\title{
Performance evaluation of the Roche Elecsys Anti-SARS-CoV-2 $S$ immunoassay
}

\author{
Elena Riester ${ }^{\mathrm{a}}$, Peter Findeisen ${ }^{\mathrm{b}}$, J. Kolja Hegel ${ }^{\mathrm{c}}$, Michael Kabesch ${ }^{\mathrm{d}}$, Andreas Ambrosch ${ }^{\mathrm{e}}$, \\ Christopher M. Rank ${ }^{\mathrm{f}}$, Florina Pessl ${ }^{\mathrm{f}}$, Tina Laengin ${ }^{\mathrm{f}}$, Christoph Niederhauser ${ }^{\mathrm{g}, \mathrm{h}, *}$ \\ ${ }^{a}$ Labor Augsburg MVZ GmbH, Augsburg, Germany \\ ${ }^{\mathrm{b}}$ MVZ Labor Limbach, Heidelberg, Germany \\ ${ }^{\mathrm{c}}$ Labor Berlin, Charité Vivantes Services GmbH, Berlin, Germany \\ ${ }^{\mathrm{d}}$ University Children's Hospital Regensburg (KUNO) at the Hospital St. Hedwig of the Order of St. John and the University Hospital, University of Regensburg, Germany \\ ${ }^{\mathrm{e}}$ Institute for Laboratory Medicine, Microbiology and Hygiene, Barmherzige Brüder Hospital, Regensburg, Germany \\ ${ }^{\mathrm{f}}$ Roche Diagnostics GmbH, Penzberg, Germany \\ ${ }^{\mathrm{g}}$ Interregionale Blood Transfusion Swiss Red Cross, Bern, Switzerland \\ ${ }^{\mathrm{h}}$ Institute for Infectious Diseases (IFIK), University of Bern, Bern, Switzerland
}

\section{A R T I C L E I N F O}

\section{Keywords:}

SARS-CoV-2

Spike protein

Nucleocapsid protein

Immunoassay

Antibodies

\begin{abstract}
A B S T R A C T
The Elecsys ${ }^{\circledR}$ Anti-SARS-CoV-2 S immunoassay (Roche Diagnostics International Ltd, Rotkreuz, Switzerland) has been developed for the detection of antibodies to the severe acute respiratory syndrome coronavirus 2 (SARSCoV-2) spike (S) protein. We evaluated the assay performance using samples from seven sites in Germany, Austria, and Switzerland. For specificity and sensitivity analyses, 7880 presumed negative pre-pandemic samples and 827 SARS-CoV-2 PCR-confirmed single or sequential samples from 272 different patients were tested, respectively. The overall specificity and sensitivity ( $\geq 14$ days post-PCR) for the Elecsys Anti-SARS-CoV-2 S immunoassay were 99.95\% (95\% confidence interval [CI]: 99.87-99.99; 7876/7880) and 97.92\% (95\% CI: 95.21-99.32; 235/240), respectively. The Elecsys Anti-SARS-CoV-2 S immunoassay had significantly higher specificity compared with the LIAISON ${ }^{\circledR}$ SARS-CoV-2 S1/S2 IgG (99.95\% [2032/2033] vs 98.82\% [2009/ 2033]), ADVIA Centaur ${ }^{\circledR}$ SARS-CoV-2 Total (100\% [928/928] vs 86.96\% [807/928]), ARCHITECT SARS-CoV-2 IgG (99.97\% [2931/2932] vs 99.69\% [2923/2932]), iFlash-SARS-CoV-2 IgM (100.00\% [928/928] vs 99.57\% [924/928]), and EUROIMMUN Anti-SARS-CoV-2 IgG (100.00\% [903/903] vs 97.45\% [880/903]) and IgA (100.00\% [895/895] vs 95.75\% [857/895]) assays. The Elecsys Anti-SARS-CoV-2 S immunoassay had significantly higher sensitivity ( $\geq 14$ days post-PCR) compared with the ARCHITECT SARS-CoV-2 IgG (98.70\% [76/77] vs $87.01 \%$ [67/77]), iFlash-SARS-CoV-2 IgG (100.00\% [76/76] vs 93.42\% [71/76]) and IgM (100.00\% [76/76] vs 35.53\% [27/76]), and EUROIMMUN Anti-SARS-CoV-2 IgG (98.26\% [113/115] vs 93.91\% [108/115]) assays. Therefore, the Elecsys Anti-SARS-CoV-2 S assay demonstrated a reliable performance across various sample populations for the detection of anti-S antibodies.
\end{abstract}

\section{Introduction}

In December 2019 a novel coronavirus emerged (Chan et al., 2020), named severe acute respiratory syndrome coronavirus 2 (SARS-CoV-2), which is the causative agent of the disease, COVID-19 (Wu et al., 2020; World Health Organization, 2020). SARS-CoV-2 is an enveloped, single-stranded RNA virus of the family Coronaviridae; its genome encodes 16 nonstructural proteins and four structural proteins: spike (S), envelope (E), membrane (M), and nucleocapsid (N) (Naqvi et al., 2020).
The most prominent protein component on the viral surface is the $S$ glycoprotein - a large transmembrane protein that assembles into trimers to form the distinctive surface spikes of coronaviruses (Walls et al., 2020; Tang et al., 2020). Each S monomer consists of two subunits, S1 and S2, which mediate receptor binding (via the receptor-binding domain [RBD] located in S1) and membrane fusion, respectively, leading to entry into host cells (Tang et al., 2020; Wrapp et al., 2020; Ou et al., 2020).

Following SARS-CoV-2 infection, the host mounts an immune response against the virus, including production of specific antibodies

\footnotetext{
* Corresponding author at: Interregionale Blood Transfusion Swiss Red Cross, Murtenstrasse 133, 3008 Bern, Switzerland.

E-mail address: Christoph.Niederhauser@itransfusion.ch (C. Niederhauser).
} 
against viral antigens (Galipeau et al., 2020). Understanding the dynamics of the antibody response to the virus is critical in establishing a relevant time window to use for serology testing (Galipeau et al., 2020). Studies into the kinetics of antibodies to SARS-CoV-2 are rapidly emerging and, both immunoglobulin $\mathrm{M}$ (IgM) and $\mathrm{G}(\mathrm{IgG})$ antibodies have been detected as early as day 1 after symptom onset (Guo et al., 2020). The chronological order of appearance and levels of IgM and IgG seems to be highly variable and often simultaneous (To et al., 2020; Long et al., 2020; Zhao et al., 2020). Several studies have observed median seroconversion at day 10-13 after symptom onset for IgM and day 12-15 for IgG, with maximum seroconversion for IgM, IgG, and total antibodies occurring at week $2-3$, week $2-4$, and around week 2 , respectively (Long et al., 2020; Zhao et al., 2020; Lou et al., 2020; Young et al., 2020).

Emergence of the COVID-19 pandemic has resulted in an urgent and unmet need to develop reliable serological tests to determine past exposure to the virus and the seroprevalence in a given population (Kontou et al., 2020). This information is crucial to support diagnosis, contact tracing, epidemiological studies, and vaccine development to enable characterization of pre-vaccination immune status and vaccine-induced immune response (Galipeau et al., 2020; Ernst et al., 2021; Zhu et al., 2020; Widge et al., 2021). There are currently 294 candidate SARS-CoV-2 vaccines in development (status August 03, 2021) (World Health Organization, 2021) and, of these, 17 are currently in early, limited, or fully approved use (status July 07, 2021) (Gavi, 2021). The majority of the vaccines in use are based on the $S$ protein, with the goal of eliciting protective neutralizing antibodies; the rest are based on whole inactivated SARS-CoV-2 (Dai and Gao, 2021; Forni and Mantovani, 2021). Serology assays are also needed for the identification of neutralizing antibodies from convalescent plasma donors (Ni et al., 2020).

The Elecsys ${ }^{\circledR}$ Anti-SARS-CoV-2 S (Roche Diagnostics International Ltd, Rotkreuz, Switzerland) is an electrochemiluminescence immunoassay (ECLIA), which has been developed for the in vitro quantitative detection of antibodies, including IgG, against the SARS-CoV-2 $S$ protein RBD in human serum and plasma (Roche Diagnostics GmbH, 2021a).

The objective of this multicenter European study was to qualitatively evaluate the specificity and sensitivity of the Elecsys Anti-SARS-CoV-2 S immunoassay using pre-pandemic samples (from routine diagnostics or blood donation) and polymerase chain reaction (PCR)-positive samples, respectively, as well as compare the performance of this quantitative test with other commercially available immunoassays in terms of specificity and sensitivity.

\section{Materials and methods}

\subsection{Study design}

The study was executed from August 17, 2020 to September 1, 2020 with samples tested at four European sites: Labor Augsburg MVZ GmbH, Augsburg, Germany; MVZ Labor Dr. Limbach \& Kollegen GbR, Heidelberg, Germany; Interregionale Blutspende SRK AG (SRK Bern), Bern, Switzerland; and Krankenhaus Barmherzige Brüder, Regensburg, Germany. Samples were collected from those four sites, as well as from three additional sites: Labor Berlin - Charité Vivantes GmbH, Berlin, Germany; Tirol Kliniken, Innsbruck, Austria; and Deutsches Rotes Kreuz Blutspendedienst West, Hagen, Germany.

Samples from Augsburg and Heidelberg included those referred to the respective study site by physicians. Heidelberg also tested samples from employees and hospitalized patients, including a subset from patients receiving dialysis. All samples provided by the study site in Berlin were collected from hospitalized patients, including a subset from patients monitored in the intensive care unit. Samples tested in Regensburg were taken from employees and pediatric patients referred to the site by physicians.
These samples were collected and tested in accordance with applicable regulations, including relevant European Union directives and regulations, and the principles of the Declaration of Helsinki. All samples from Augsburg, Heidelberg, Berlin and Hagen were anonymized. A statement was obtained from the Ethics Committee (EC) of the Landesärztekammer Bayern confirming that there are no objections to the use of anonymized leftover samples. From the EC at the study site in Bern (Switzerland) a waiver was received and from the internal EC at the study site in Innsbruck (Austria) an approval was received. For Regensburg (Germany), EC approvals were already in place, amendments were submitted to notify the EC about Elecsys Anti-SARS-CoV-2 $\mathrm{S}$ testing. At Augsburg, Heidelberg, and Bern the assays were performed on the cobas e 801 analyzer (Roche Diagnostics International Ltd, Rotkreuz, Switzerland), whereas at Regensburg the assays were performed on the cobas e 601 analyzer (Roche Diagnostics International Ltd, Rotkreuz, Switzerland). To assess the proper function of cobas e instruments, applications and reagents, a familiarization experiment was performed with quality control material and successfully passed at all measuring sites. Sites were requested to run quality controls after every calibration, for each reagent kit, and at least once every $24 \mathrm{~h}$, and to proceed only if the recovery was within the acceptance criteria.

\subsection{Serum and plasma samples}

Anonymized frozen, residual serum or plasma samples $(\geq 300 \mu \mathrm{l}$ volume) from blood donation centers or routine laboratory diagnostics, as well as banked samples, were used for this study. For specificity analysis of the Elecsys Anti-SARS-CoV-2 S assay, 7880 samples (5056 blood donor and 2824 diagnostic routine samples) that were collected before the COVID-19 pandemic in October 2019, and therefore presumed to be negative for SARS-CoV-2, were tested. The diagnostic routine cohort included samples from women attending pregnancy screening and from pediatrics. For the sensitivity analysis of the Elecsys Anti-SARS-CoV-2 S assay, 827 PCR-confirmed single or sequential samples from 272 different patients, with known time difference between blood draw and positive PCR test, were tested. Of these presumed negative and PCR-confirmed samples, 7903 were tested on the commercially available Elecsys ${ }^{\circledR}$ Anti-SARS-CoV-2 assay (Roche Diagnostics International Ltd, Rotkreuz, Switzerland) (Roche Diagnostics $\mathrm{GmbH}, 2021 \mathrm{~b}$ ). Additionally, a number of these samples were tested on other commercially available assays: LIAISON ${ }^{\circledR}$ SARS-CoV-2 S1/S2 IgG (DiaSorin, Saluggia, Italy) (DiaSorin, 2020), 2052 samples; EUROIMMUN Anti-SARS-CoV-2 IgG (EUROIMMUN, 2020a) and immunoglobulin A (IgA) assays (EUROIMMUN, Lübeck, Germany) (EUROIMMUN, 2020b), 1618 and 1624 samples, respectively; ARCHITECT SARS-CoV-2 IgG (Abbott Laboratories, Abbott Park, Illinois, USA) (Abbott, 2021), 3068 samples; ADVIA Centaur ${ }^{\circledR}$ SARS-CoV-2 Total (Siemens, Tarrytown, New York, USA) (Siemens, 2020), 1064 samples; iFlash-SARS-CoV-2 IgG and IgM assays (YHLO Biotech Co., Ltd, Shenzhen, China) (Shenzhen YHLO Biotech Co Ltd., 2020), both 1062 samples.

\subsection{Elecsys Anti-SARS-CoV-2 S assay}

The Elecsys Anti-SARS-CoV-2 S immunoassay is a quantitative ECLIA that detects high-affinity antibodies to the SARS-CoV-2 S protein RBD and has a low risk of detecting weakly cross-reactive and unspecific antibodies. Results are automatically reported as the analyte concentration of each sample in $\mathrm{U} / \mathrm{mL}$, with $<0.80 \mathrm{U} / \mathrm{mL}$ interpreted as negative for anti-SARS-CoV- $2 \mathrm{~S}$ antibodies and $\geq 0.80 \mathrm{U} / \mathrm{mL}$ interpreted as positive for anti-SARS-CoV-2 $\mathrm{S}$ antibodies (Roche Diagnostics $\mathrm{GmbH}$. Elecsys Anti-SARS-CoV-2 S assay method sheet. 2020; version 2.0) (Roche Diagnostics GmbH, 2021c). 


\subsection{Comparator assays}

Specimens were analyzed using eight comparator immunoassays according to the manufacturer's instructions. Interpretation of results was performed according to the manufacturer's instructions.

The Elecsys Anti-SARS-CoV-2 assay is an ECLIA for the in vitro qualitative detection of antibodies, including IgG, against SARS-CoV-2, using a recombinant protein representing the $\mathrm{N}$ antigen (Roche Diagnostics $\mathrm{GmbH}, 2021 b)$. Results are automatically calculated in the form of a cutoff index (COI), with values $<1.0$ interpreted as non-reactive (negative) for anti-SARS-CoV- $2 \mathrm{~N}$ antibodies and $\geq 1.0$ as reactive (positive) for anti-SARS-CoV-2 N antibodies (Roche Diagnostics GmbH, 2021b).

The LIAISON SARS-CoV-2 S1/S2 IgG assay is an indirect chemiluminescence immunoassay (CLIA) for the quantitative detection of IgG anti-S1 and IgG anti-S2 antibodies to SARS-CoV-2 (DiaSorin, 2020). Results are automatically calculated, with antibody concentrations expressed as arbitrary units (AU/mL). Concentrations of $<12.0 \mathrm{AU} / \mathrm{mL}$ are interpreted as negative, $\geq 12.0$ to $<15.0 \mathrm{AU} / \mathrm{mL}$ are interpreted as equivocal, and $\geq 15.0 \mathrm{AU} / \mathrm{mL}$ are interpreted as positive (DiaSorin, 2020). Equivocal values are referred to as 'gray zone' results.

The EUROIMMUN Anti-SARS-CoV-2 IgG and IgA assays are separate enzyme-linked immunosorbent assays (ELISAs) that detect IgG or IgA anti-S1 antibodies to SARS-CoV-2 (EUROIMMUN, 2020a, b). Results are evaluated semi-quantitatively by calculation of a ratio in which the absorbance values of the controls or patient samples are related to the absorbance value of the calibrator (EUROIMMUN, 2020a, b). For both assays, ratio results $<0.8$ are interpreted as negative, $\geq 0.8$ to $<1.1$ are borderline, and $\geq 1.1$ are positive (EUROIMMUN, 2020a, b). Borderline values are referred to as 'gray zone' results.

The ARCHITECT SARS-CoV-2 IgG assay is a chemiluminescent microparticle immunoassay (CMIA) used for the qualitative detection of IgG antibodies against the $\mathrm{N}$ antigen (Abbott, 2021). Results are expressed in signal-to-cutoff (S/CO) values, with $<1.4$ results interpreted as negative and $\geq 1.4$ results interpreted as positive (Abbott, 2021).

The ADVIA Centaur SARS-CoV-2 Total assay is a CLIA intended for the qualitative detection of antibodies against the RBD of the $S 1$ protein (Siemens, 2020). Results are reported in index values, with $<1.0$ interpreted as non-reactive (negative) for anti-SARS-CoV-2 antibodies and $\geq 1.0$ interpreted as reactive (positive) for anti-SARS-CoV-2 antibodies (Siemens, 2020).

The iFlash-SARS-CoV-2 IgM and IgG assays (Shenzhen YHLO Biotech Co Ltd., 2020) are separate CLIAs used for the qualitative detection of IgM or IgG against the $S$ and N proteins. The iFlash system automatically calculates the analytic concentration of each sample, with $<10 \mathrm{AU} / \mathrm{mL}$ interpreted as non-reactive and $\geq 10 \mathrm{AU} / \mathrm{mL}$ interpreted as reactive for anti-SARS-CoV-2 IgM or IgG antibodies.

\subsection{Statistical analysis}

Sample size estimations for specificity and sensitivity analyses were based on formulae proposed previously (Hajian-Tilaki, 2014). Assuming specificities between 0.998 and 0.999 and a sensitivity of 0.999 , samples sizes of 1698-20964 and 32-50 respectively, would be required to obtain a significance level of 0.05 and a power of 0.8 . For specificity and sensitivity calculations, point estimates and two-sided $95 \%$ confidence intervals (CIs) using the exact method were computed employing $\mathrm{R}$ version 3.4.0 (R Core Team, 2017). In the sensitivity evaluation, assay results were assigned to the respective week after positive PCR result. In the comparison with other commercially available assays, only samples with paired measurements were included in the respective analyses. For the differences in estimated specificities and sensitivities between Elecsys Anti-SARS-CoV-2 S assay and the comparator assays, two-sided 95\% Wald CIs were calculated as previously recommended (Wenzel and Zapf, 2013). If these CIs did not include zero, differences were considered as statistically significant.

\section{Results}

\subsection{Overall performance of the elecsys Anti-SARS-CoV-2 $S$ assay}

\subsubsection{Specificity in different target cohorts}

Specificity of the Elecsys Anti-SARS-CoV-2 S assay was evaluated at three European sites (with samples from five European sites) using 7880 evaluable residual samples from blood donors and routine diagnostic testing; all of which were collected before October 2019 and presumed negative for SARS-CoV-2 antibodies. The overall specificity for all samples was 99.95\% (95\% CI: 99.87-99.99 [7876/7880]) (Table 1). There were four samples with reactive results of $1.790 \mathrm{U} / \mathrm{mL}, 0.900 \mathrm{U} /$ $\mathrm{mL}, 0.870 \mathrm{U} / \mathrm{mL}$, and $1.130 \mathrm{U} / \mathrm{mL}$. Three of these reactive samples were from blood donor samples, of which one was collected in March 2016 (influenza season) at Innsbruck, Austria and two were collected in July/ August 2018 (outside influenza season) at Bern, Switzerland (Table 1). There was no statistically significant difference in specificity between blood donor samples collected during or outside influenza season. The other reactive sample was from the pregnancy screening cohort in Augsburg (Table 1).

\subsubsection{Sensitivity in different target cohorts}

In total, 827 single and sequential samples from 272 SARS-CoV-2 PCR-confirmed patients were evaluated at three European sites (with samples from four European sites). The time span of samples collected after positive PCR was between day 0 and day 120. For subjects with sequential blood draws with more than one sample per time interval, only the result of the last blood draw per given time interval was used for the respective sensitivity calculation. The sensitivity of the Elecsys AntiSARS-CoV-2 S assay $\geq 14$ days post-PCR $(n=240)$ was $97.92 \%$ ( $95 \%$ CI: 95.21-99.32 [235/240]) (Table 2). The resulting site-specific sensitivities for Augsburg, Berlin, Heidelberg, and Regensburg samples collected $\geq 14$ days post-PCR confirmation were $100.00 \%$ (95\% CI: 95.89-100.00), 100.00\% (95\% CI: 91.40-100.00), 98.72\% (95\% CI: 93.06-99.97\%), and 87.88\% (95\% CI: 71.80-96.60), respectively.

\subsubsection{Determination of seroconversions}

For all subjects with at least two sequential blood draws, trajectories were plotted to determine antibody titer development from day 0-78 post-PCR-positive test (Fig. 1). Most trajectories showed a rapid increase

Table 1

Specificity results for the Elecsys Anti-SARS-CoV-2 S assay.

\begin{tabular}{|c|c|c|c|}
\hline \multicolumn{2}{|c|}{ Sample cohort } & $\begin{array}{l}\text { Reactive } \\
\text { samples/ number } \\
\text { of samples }\end{array}$ & $\begin{array}{l}\text { Specificity ( } 95 \% \\
\text { confidence intervals, 2- } \\
\text { sided) }\end{array}$ \\
\hline \multicolumn{4}{|c|}{ Blood donors } \\
\hline \multirow{4}{*}{$\begin{array}{l}\text { Origin / } \\
\text { season }\end{array}$} & $\begin{array}{l}\text { Innsbruck / } \\
\text { influenza season }\end{array}$ & $1 / 1050$ & $99.90 \%(99.47-100.00)$ \\
\hline & $\begin{array}{l}\text { Hagen / no } \\
\text { seasonal selection }\end{array}$ & $0 / 955$ & $100.00 \%(99.61-100.00)$ \\
\hline & $\begin{array}{l}\text { Bern / outside } \\
\text { influenza season }\end{array}$ & $2 / 2000$ & 99.90\% (99.64-99.99) \\
\hline & $\begin{array}{l}\text { Bern / influenza } \\
\text { season }\end{array}$ & $0 / 1051$ & $100.00 \%(99.65-100.00)$ \\
\hline \multirow{2}{*}{\multicolumn{2}{|c|}{$\begin{array}{l}\text { Total blood donors } \\
\text { Diagnostic routine }\end{array}$}} & $3 / 5056$ & 99.94\% (99.83-99.99) \\
\hline & & \multicolumn{2}{|c|}{ Diagnostic routine } \\
\hline \multirow{5}{*}{$\begin{array}{r}\text { Origin / } \\
\text { cohort }\end{array}$} & $\begin{array}{l}\text { Augsburg / } \\
\text { diagnostic routine }\end{array}$ & $0 / 400$ & $100.00 \%(99.08-100.00)$ \\
\hline & $\begin{array}{l}\text { Augsburg / } \\
\text { pregnancy }\end{array}$ & $1 / 1496$ & $99.93 \%$ (99.63-100.00) \\
\hline & screening & & \\
\hline & $\begin{array}{l}\text { Heidelberg / } \\
\text { pregnancy } \\
\text { screening }\end{array}$ & $0 / 737$ & $100.00 \%(99.50-100.00)$ \\
\hline & $\begin{array}{l}\text { Heidelberg / } \\
\text { pediatric samples }\end{array}$ & $0 / 191$ & $100.00 \%(98.09-100.00)$ \\
\hline \multicolumn{2}{|c|}{ Total diagnostic routine } & $1 / 2824$ & $99.96 \%(99.80-100.00)$ \\
\hline \multicolumn{2}{|c|}{ Overall (all samples) } & $4 / 7880$ & 99.95\% (99.87-99.99) \\
\hline
\end{tabular}


Table 2

Overall sensitivity results for the Elecsys Anti-SARS-CoV-2 S assay.

\begin{tabular}{lll}
\hline $\begin{array}{l}\text { Days post-PCR- } \\
\text { positive test }\end{array}$ & $\begin{array}{l}\text { Non-reactive samples/ } \\
\text { number of samples }\end{array}$ & $\begin{array}{l}\text { Sensitivity (95\% confidence } \\
\text { intervals, 2-sided) }\end{array}$ \\
\hline 0 to 6 & $19 / 44$ & $56.82 \%(41.03-71.65)$ \\
7 to 13 & $7 / 49$ & $85.71 \%(72.76-94.06)$ \\
14 to 20 & $1 / 47$ & $97.87 \%(88.71-99.95)$ \\
21 to 27 & $0 / 58$ & $100.00 \%(93.84-100.00)$ \\
28 to 34 & $0 / 43$ & $100.00 \%(91.78-100.00)$ \\
35 to 41 & $1 / 57$ & $98.25 \%(90.61-99.96)$ \\
42 to 48 & $0 / 47$ & $100.00 \%(92.45-100.00)$ \\
49 to 55 & $4 / 39$ & $89.74 \%(75.78-97.13)$ \\
56 to 62 & $0 / 33$ & $100.00 \%(89.42-100.00)$ \\
$\geq 63$ (up to 120$)$ & $0 / 21$ & $100.00 \%(83.89-100.00)$ \\
All samples $\geq \mathbf{1 4}$ & $\mathbf{5 / 2 4 0}$ & $\mathbf{9 7 . 9 2 \% ( 9 5 . 2 1 - 9 9 . 3 2 )}$ \\
$\quad$ (up to 120$)^{\mathrm{a}}$ & &
\end{tabular}

PCR, polymerase chain reaction.

a Only the last sample taken $\geq 14$ days post-PCR of each patient is included in the sensitivity calculation.

in antibody titer and no considerable decline of antibody titer was seen for the early and later blood draws. Once detected reactive, none of the subsequent samples drawn per subject showed a decline of titer below the cutoff.

\subsection{Comparison with Elecsys Anti-SARS-CoV-2 assay}

A direct method comparison between the Elecsys Anti-SARS-CoV-2 S assay and the commercially available Elecsys Anti-SARS-CoV-2 assay was performed. This included a total of 7903 samples comprising both confirmed positive samples from sensitivity testing and presumed positive samples with at least one positive antibody result $(n=1011)$, as well as presumed negative samples from specificity testing cohort samples $(n=6892: n=4068$ blood donors; $n=2824$ routine diagnostic). For all samples, the overall percent agreement (OPA) between the Elecsys Anti-SARS-CoV-2 S assay and the Elecsys Anti-SARS-CoV-2 assay was 99.30\% (95\% CI: 99.10-99.48) (Supplemental Table 1).

\subsection{Comparison with other commercially available assays}

The performance of the Elecsys Anti-SARS-CoV-2 S immunoassay was compared with seven other commercially available SARS-CoV-2 assays, and sensitivity and specificity results, along with percent agreement, were recorded. Information regarding the different assays, including the technique, target, and Ig measured can be found in Supplemental Table 2.
The OPA between the Elecsys Anti-SARS-CoV-2 S assay and other comparator tests was recorded (Supplemental Table 2). The Elecsys Anti-SARS-CoV-2 $\mathrm{S}$ test had the highest OPA with the ARCHITECT SARSCoV-2 IgG (N-assay), at 99.19\% (95\% CI: 98.80-99.47), and the lowest OPA with the ADVIA Centaur SARS-CoV-2 Total (S-assay), at $88.25 \%$ (95\% CI: 86.16-90.13) (Supplemental Table 3).

\subsubsection{Specificity}

The specificity of the Elecsys Anti-SARS-CoV-2 S assay was comparable or higher than the specificity of all tested comparator assays (Table 3). The specificity of the Elecsys Anti-SARS-CoV-2 S test was significantly higher compared with the LIAISON SARS-CoV-2 S1/S2 IgG (99.95\% [2032/2033] vs 98.82\% [2009/2033]), ADVIA Centaur SARSCoV-2 Total (100\% [928/928] vs $86.96 \%$ [807/928]), ARCHITECT SARS-CoV-2 IgG (99.97\% [2931/2932] vs 99.69\% [2923/2932]), iFlash-SARS-CoV-2 IgM (100.00\% [928/928] vs 99.57\% [924/928]), and EUROIMMUN Anti-SARS-CoV-2 IgG (100.00\% [903/903] vs 97.45\% [880/903]) and IgA (100.00\% [895/895] vs 95.75\% [857/ 895]) assays (Table 3, Supplemental Table 4A). No statistically significant difference was observed between the specificity of the Elecsys AntiSARS-CoV-2 $\mathrm{S}$ assay compared with the iFlash-SARS-CoV-2 IgG assay, which was $100.00 \%(928 / 928)$ for both assays (Table 3, Supplemental Table 4A).

\subsubsection{Sensitivity}

The sensitivity of the Elecsys Anti-SARS-CoV-2 S assay for detecting seropositive results was compared with six comparator assays; analysis compared with the LIAISON SARS-CoV-2 S1/S2 IgG test could not be performed due to a small sample size. Sensitivity was recorded for samples collected between $0-6,7-13$, and $\geq 14$ days post-PCR-positive test (Table 4). The EUROIMMUN Anti-SARS-CoV-2 IgA assay showed a higher sensitivity in the $0-6(59.09 \%$ [26/44] vs $56.82 \%$ [25/44]) and 7-13 days (91.84\% [45/49] vs $85.71 \%$ [42/49]) post-PCR time intervals and a lower sensitivity in the $\geq 14$ days $(97.46 \%$ [115/118] vs $99.15 \%$ [117/118]) post-PCR time interval compared with the Elecsys AntiSARS-CoV-2 S assay (Table 4). The sensitivity of the Elecsys AntiSARS-CoV-2 $S$ assay at detecting antibodies $\geq 14$ days post-PCR was significantly higher compared with the ARCHITECT SARS-CoV-2 IgG $(98.70 \%[76 / 77]$ vs $87.01 \%$ [67/77]), iFlash-SARS-CoV-2 IgG $(100.00 \%[76 / 76]$ vs $93.42 \%$ [71/76]) and IgM (100.00\% [76/76] vs $35.53 \%$ [27/76]), and EUROIMMUN Anti-SARS-CoV-2 IgG (98.26\% [113/115] vs 93.91\% [108/115]) assays (Table 4, Supplemental Table 4B).

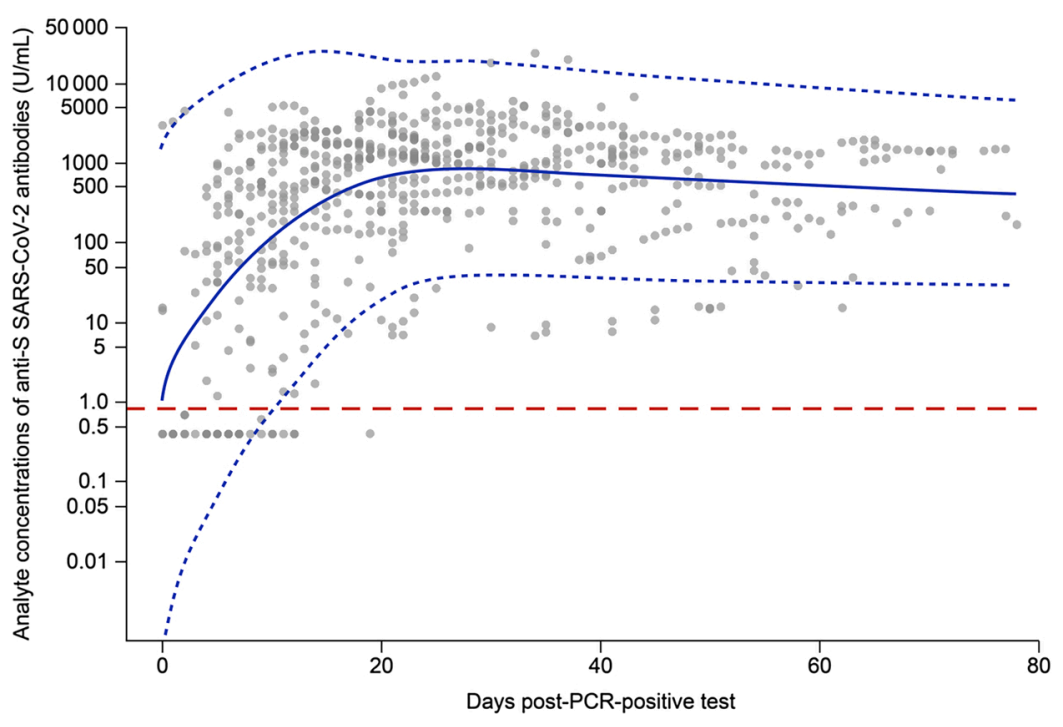

Fig. 1. Longitudinal antibody titers of all subjects. Concentration of anti-S SARS-CoV-2 antibodies, as measured by the Elecsys Anti-S SARS-CoV-2 S immunoassay, over time (days 0 to 78) in sequential samples from all study sites. Each grey circle represents a different data point, with darker circles representing overlapping data points. The solid blue line represents the combined curve, and the blue dashed lines represent the upper and lower confidence limits. The red dashed line indicates the assay cutoff limit $(0.80 \mathrm{U} / \mathrm{mL})$. 
Table 3

Specificity of the Elecsys Anti-SARS-CoV-2 S assay and other comparator assays.

\begin{tabular}{|c|c|c|}
\hline Assay & Reactive samples/ number of samples & Specificity ( $95 \%$ confidence intervals, 2-sided) \\
\hline \multicolumn{3}{|l|}{ Comparison with anti-S assays } \\
\hline LIAISON SARS-CoV-2 S1/S2 IgG (GZ excl.) ${ }^{\mathrm{a}}$ & $24 / 2033$ & $98.82 \%(98.25-99.24)$ \\
\hline Elecsys Anti-SARS-CoV-2 S & $1 / 2033$ & $99.95 \%(99.73-100.00)$ \\
\hline LIAISON SARS-CoV-2 S1/S2 IgG (GZ+) ${ }^{\mathrm{a}}$ & $31 / 2040$ & $98.48 \%(97.85-98.97)$ \\
\hline Elecsys Anti-SARS-CoV-2 S & $1 / 2040$ & $99.95 \%(99.73-100.00)$ \\
\hline ADVIA Centaur SARS-CoV-2 Total & $121 / 928$ & $86.96 \%(84.62-89.06)$ \\
\hline Elecsys Anti-SARS-CoV-2 S & $0 / 928$ & $100.00 \%(99.60-100.00)$ \\
\hline EUROIMMUN Anti-SARS-CoV-2 IgG (GZ excl.) & $23 / 903$ & $97.45 \%(96.20-98.38)$ \\
\hline Elecsys Anti-SARS-CoV-2 S & $0 / 903$ & $100.00 \%(99.59-100.00)$ \\
\hline EUROIMMUN Anti-SARS-CoV-2 IgG (GZ+) & $44 / 924$ & $95.24 \%(93.66-96.52)$ \\
\hline Elecsys Anti-SARS-CoV-2 S & $0 / 924$ & $100.00 \%(99.60-100.00)$ \\
\hline EUROIMMUN Anti-SARS-CoV-2 IgA (GZ excl.) & $38 / 895$ & $95.75 \%(94.22-96.98)$ \\
\hline Elecsys Anti-SARS-CoV-2 S & $0 / 895$ & $100.00 \%(99.59-100.00)$ \\
\hline EUROIMMUN Anti-SARS-CoV-2 IgA (GZ+) & $71 / 928$ & $92.35 \%(90.45-93.98)$ \\
\hline Elecsys Anti-SARS-CoV-2 S & $0 / 928$ & $100.00 \%(99.60-100.00)$ \\
\hline \multicolumn{3}{|l|}{ Comparison with anti-N assay } \\
\hline ARCHITECT SARS-CoV-2 IgG & $9 / 2932$ & $99.69 \%(99.42-99.86)$ \\
\hline Elecsys Anti-SARS-CoV-2 S & $1 / 2932$ & $99.97 \%(99.81-100.00)$ \\
\hline \multicolumn{3}{|l|}{ Comparison with anti-S and $-\mathrm{N}$ assays } \\
\hline iFlash-SARS-CoV-2 IgG & $0 / 928$ & $100.00 \%(99.60-100.00)$ \\
\hline Elecsys Anti-SARS-CoV-2 S & $0 / 928$ & $100.00 \%(99.60-100.00)$ \\
\hline iFlash-SARS-CoV-2 IgM & $4 / 928$ & $99.57 \%(98.90-99.88)$ \\
\hline Elecsys Anti-SARS-CoV-2 S & $0 / 928$ & $100.00 \%(99.60-100.00)$ \\
\hline
\end{tabular}

GZ, gray zone.

a For antibody assays with a gray zone, two calculations were performed. In the first calculation, all gray zone results were excluded from the analysis (GZ excl.) and in the second calculation these results were interpreted as reactive $(\mathrm{GZ}+)$.

\section{Discussion}

Due to the COVID-19 pandemic, there is a pressing need to develop highly specific and sensitive serology tests to assist with the diagnosis of, and to reveal past exposure to, the SARS-CoV-2 virus (Kontou et al., 2020), as well as to support the development of vaccines through distinguishing natural infection-induced immunity from vaccine-induced immunity (Galipeau et al., 2020; Ernst et al., 2021). This was the first multicenter study to demonstrate the performance of the automated Elecsys Anti-SARS-CoV-2 S immunoassay, which detects antibodies against the SARS-CoV-2 $S$ protein RBD. Antibodies against the RBD have previously been shown to correlate strongly with protective neutralizing antibodies (Premkumar et al., 2020).

The results from our study revealed that the Elecsys Anti-SARS-CoV$2 \mathrm{~S}$ immunoassay displays a robust performance under routine conditions at multiple sites in Europe, with a very high specificity $(99.95 \%$ [7876/7880]) and sensitivity (97.92\% [235/240]) for the detection of anti-S antibodies. The point estimates for specificity and sensitivity are comparable to the values reported in the package insert of the Elecsys Anti-SARS-CoV-2 S assay (99.98\% and 98.8\%, respectively) (Roche Diagnostics GmbH, 2021a). In addition, the Elecsys Anti-SARS-CoV-2 S assay showed a performance comparable with the commercially available Elecsys Anti-SARS-CoV-2 (N-assay), with 95\% CIs that overlap (99.69-99.88\% for specificity and $97.0-100 \%$ for sensitivity); both assays had a very high overall percent agreement. The Elecsys Anti-SARS-CoV-2 assay has a previously reported specificity and sensitivity $\geq 14$ days post-confirmation of $99.8 \%$ and $99.5 \%$, respectively (Muench et al., 2020). These data indicate that the Elecsys Anti-SARS-CoV-2 S assay can be utilized to meet the current need for anti-SARS-CoV-2 antibody testing, alongside or in place of the Elecsys Anti-SARS-CoV-2 (N) assay, depending on the study objectives.

The overall specificity of $>99.9 \%$ determined in this study demonstrated that the Elecsys Anti-SARS-CoV-2 S is a highly specific assay for the detection of antibodies against SARS-CoV-2. Notably, this analysis included 2424 samples from pregnant women and pediatric populations. The availability of an accurate SARS-CoV-2 serology assay is particularly important for the pregnant population, considering the changes in the immune system that occur during pregnancy, which may increase the woman's susceptibility to severe infection (Dashraath et al., 2020). Additionally, an antibody assay with a high specificity is imperative to reduce the risk of false-positive results, which may inaccurately indicate a past SARS-CoV-2 infection (Farnsworth and Anderson, 2020). Our study did not assess the specificity of the Elecsys Anti-SARS-CoV-2 S assay using samples from individuals with other respiratory infections. However, this assay has been previously tested using 1468 samples containing potentially cross-reactive samples collected before October 2019 , and $100 \%$ specificity was obtained with no cross-reactivity (Roche Diagnostics $\mathrm{GmbH}, 2021 \mathrm{c}$ ). These samples were from individuals confirmed to be infected with MERS-CoV, common cold coronaviruses, or other respiratory infections (Roche Diagnostics GmbH, 2021c).

The Elecsys Anti-SARS-CoV-2 S immunoassay demonstrated good specificity (99.95-100.00\%) and sensitivity at $\geq 14$ days post-PCRpositive test (98.26-100.00\%) in direct comparison with other commercially available assays; both performance measurements were equal to or greater than those for other evaluated comparator assays. These other assays have also been assessed in previous studies (Kontou et al., 2020; Meyer et al., 2020; Kohmer et al., 2020; Okba et al., 2020; Ocmant et al., 2021). However, it is important to note that, for a direct comparison of sensitivity, the available assays differ with respect to assay designs (e.g. antibody classes used) as well as the targets (anti-N and anti-S) that they detect.

A multicenter comparison of seven serology assays, including the Elecsys Anti-SARS-CoV-2 assay, revealed a subpopulation of PCRconfirmed SARS-CoV-2 individuals who were persistently seronegative, which represents a proportion of patients who may be at risk for reinfection (Oved et al., 2020). Within the group of PCR-confirmed 
Table 4

Sensitivity of the Elecsys Anti-SARS-CoV-2 S assay and other comparator assays.

\begin{tabular}{|c|c|c|c|}
\hline Assay & Days post-PCR-positive test & Non-reactive samples/ number of samples & Sensitivity ( $95 \%$ confidence intervals, 2 -sided) \\
\hline \multicolumn{4}{|l|}{ Comparison with anti-S assays } \\
\hline \multirow[t]{2}{*}{ ADVIA Centaur SARS-CoV-2 Total } & \multirow{2}{*}{$0-6$} & $8 / 18$ & $55.56 \%(30.76-78.47)$ \\
\hline & & $8 / 18$ & $55.56 \%(30.76-78.47)$ \\
\hline \multirow{4}{*}{ Elecsys Anti-SARS-CoV-2 S } & \multirow{2}{*}{$7-13$} & $2 / 11$ & $81.82 \%(48.22-97.72)$ \\
\hline & & $2 / 11$ & $81.82 \%(48.22-97.72)$ \\
\hline & \multirow{2}{*}{$\geq 14$} & $4 / 77$ & $94.81 \%(87.23-98.57)$ \\
\hline & & $1 / 77$ & $98.70 \%(92.98-99.97)$ \\
\hline \multirow[t]{3}{*}{$\begin{array}{l}\text { EUROIMMUN Anti-SARS-CoV-2 IgG } \\
(\text { (GZ excl. })^{\mathrm{a}}\end{array}$} & \multirow{2}{*}{$0-6$} & $21 / 43$ & $51.16 \%(35.46-66.69)$ \\
\hline & & $18 / 43$ & $58.14 \%(42.13-72.99)$ \\
\hline & \multirow{2}{*}{$7-13$} & $7 / 45$ & $84.44 \%(70.54-93.51)$ \\
\hline \multirow[t]{3}{*}{ Elecsys Anti-SARS-CoV-2 S } & & $7 / 45$ & $84.44 \%(70.54-93.51)$ \\
\hline & \multirow{2}{*}{$\geq 14$} & $7 / 115$ & $93.91 \%(87.86-97.52)$ \\
\hline & & $2 / 115$ & $98.26 \%(93.86-99.79)$ \\
\hline \multirow[t]{3}{*}{$\begin{array}{l}\text { EUROIMMUN Anti-SARS-CoV-2 IgG } \\
(\mathrm{GZ}+)^{\mathrm{a}}\end{array}$} & \multirow{2}{*}{$0-6$} & $21 / 44$ & $52.27 \%(36.69-67.54)$ \\
\hline & & $19 / 44$ & $56.82 \%(41.03-71.65)$ \\
\hline & \multirow{2}{*}{$7-13$} & $7 / 49$ & $85.71 \%(72.76-94.06)$ \\
\hline \multirow[t]{3}{*}{ Elecsys Anti-SARS-CoV-2 S } & & $7 / 49$ & $85.71 \%(72.76-94.06)$ \\
\hline & \multirow{2}{*}{$\geq 14$} & $6 / 119$ & $94.96 \%(89.35-98.13)$ \\
\hline & & $1 / 119$ & $99.16 \%(95.41-99.98)$ \\
\hline \multirow[t]{3}{*}{$\begin{array}{l}\text { EUROIMMUN Anti-SARS-CoV-2 IgA } \\
\text { (GZ excl.) }\end{array}$} & \multirow{2}{*}{$0-6$} & $18 / 44$ & $59.09 \%(43.25-73.66)$ \\
\hline & & $19 / 44$ & $56.82 \%(41.03-71.65)$ \\
\hline & \multirow{2}{*}{$7-13$} & $4 / 49$ & $91.84 \%(80.40-97.73)$ \\
\hline \multirow[t]{3}{*}{ Elecsys Anti-SARS-CoV-2 S } & & $7 / 49$ & $85.71 \%(72.76-94.06)$ \\
\hline & \multirow{2}{*}{$\geq 14$} & $3 / 118$ & $97.46 \%(92.75-99.47)$ \\
\hline & & $1 / 118$ & $99.15 \%$ (95.37-99.98) \\
\hline $\begin{array}{l}\text { EUROIMMUN Anti-SARS-CoV-2 IgA } \\
(\mathrm{GZ}+)\end{array}$ & $0-6$ & $18 / 44$ & $59.09 \%(43.25-73.66)$ \\
\hline & & $19 / 44$ & $56.82 \%(41.03-71.65)$ \\
\hline & & $4 / 49$ & $91.84 \%(80.40-97.73)$ \\
\hline Elecsys Anti-SARS-CoV-2 S & $7-13$ & $7 / 49$ & $85.71 \%(72.76-94.06)$ \\
\hline & & $3 / 121$ & $97.52 \%(92.93-99.49)$ \\
\hline & $\geq 14$ & $1 / 121$ & $99.17 \%(95.48-99.98)$ \\
\hline Comparison with anti-N assay & & & \\
\hline ARCHITECT SARS-CoV-2 IgG & & $9 / 18$ & $50.00 \%(26.02-73.98)$ \\
\hline & $0-6$ & $8 / 18$ & $55.56 \%(30.76-78.47)$ \\
\hline & & $2 / 11$ & $81.82 \%(48.22-97.72)$ \\
\hline Elecsys Anti-SARS-CoV-2 S & $7-13$ & $2 / 11$ & $81.82 \%(48.22-97.72)$ \\
\hline & $>14$ & $10 / 77$ & $87.01 \%(77.41-93.59)$ \\
\hline & $\geq 14$ & $1 / 77$ & $98.70 \%(92.98-99.97)$ \\
\hline Comparison with anti-S and $-\mathrm{N}$ assa & & & \\
\hline iFlash-SARS-CoV-2 IgG & & $8 / 17$ & $52.94 \%(27.81-77.02)$ \\
\hline & 0-6 & $7 / 17$ & $58.82 \%(32.92-81.56)$ \\
\hline & 7-13 & $2 / 11$ & $81.82 \%(48.22-97.72)$ \\
\hline Elecsys Anti-SARS-CoV-2 S & $7-13$ & $2 / 11$ & $81.82 \%(48.22-97.72)$ \\
\hline & $>14$ & $5 / 76$ & $93.42 \%(85.31-97.83)$ \\
\hline & & $0 / 76$ & $100.00 \%(95.26-100.00)$ \\
\hline iFlash-SARS-CoV-2 IgM & $0-6$ & $11 / 17$ & $35.29 \%(14.21-61.67)$ \\
\hline & $0-6$ & $7 / 17$ & $58.82 \%(32.92-81.56)$ \\
\hline & & $9 / 11$ & $18.18 \%(2.28-51.78)$ \\
\hline Elecsys Anti-SARS-CoV-2 S & $7-13$ & $2 / 11$ & $81.82 \%(48.22-97.72)$ \\
\hline & & $49 / 76$ & $35.53 \%(24.88-47.34)$ \\
\hline & $\geq 14$ & $0 / 76$ & $100.00 \%(95.26-100.00)$ \\
\hline
\end{tabular}

GZ, gray zone.

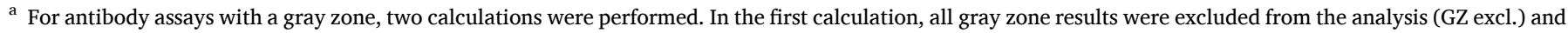
in the second calculation these results were interpreted as reactive (GZ+).

samples in our study, for which there was at least one $\mathrm{S}$ and one $\mathrm{N}$ antibody result from another comparator assay, there were samples from five patients all of which had a reactive Elecsys Anti-SARS-CoV-2 S ECLIA result, a reactive or 'gray zone' EUROIMMUN ELISA result and a reactive ADVIA Centaur CLIA result (S-assays), and a non-reactive Elecsys Anti-SARS-CoV-2 ECLIA and ARCHITECT SARS-CoV-2 IgG
CMIA result (N-assays). There was only one sample that was commonly non-reactive in all S-based assays and reactive in all N-based assays. However, the blood draw was taken very early, on the same day as the PCR was done, and the follow-up sample was reactive with the Elecsys Anti-SARS-CoV-2 S assay. Data indicating differences in the kinetics of serology assays are ambiguous and there was little difference in the 
timing of these responses (Kohmer et al., 2020; Wang et al., 2020; Van Elslande et al., 2020; Johnson et al., 2020; Burbelo et al., 2020). However, differences between $\mathrm{N}$ - and S- antigen-based assays should be taken into consideration when interpreting results.

A major strength of this study is the large cohort of presumed negative samples collected before the COVID-19 pandemic from multiple sites that were used to determine the specificity of the assay, as well as the multiple method comparison analyses performed and the various population cohorts used, with samples from pediatrics and from pregnant women. Further studies should be performed to determine the sensitivity of the Elecsys Anti-SARS-CoV-2 S immunoassay on a larger sample group.

The Elecsys Anti-SARS-CoV-2 S assay could be used to support studies requiring quantification of antibody responses and vaccine efficacy studies and may be particularly useful due to the majority of vaccines being based on the $S$ protein. Previous studies have utilized the Elecsys Anti-SARS-CoV-2 S immunoassay to determine the immune response to vaccination in various populations (Salvagno et al., 2021; Kennedy et al., 2021; Herishanu et al., 2021; Cavalcanti et al., 2021; Callegaro et al., 2021; Seyahi et al., 2021), as well as to quantify antibody levels (Rubio-Acero et al., 2021; Resman Rus et al., 2021), although further evaluation is necessary to assess the performance of the Elecsys Anti-SARS-CoV-2 S immunoassay for these specific purposes as assessing vaccine efficacy is currently outside of the intended use of the assay.

\section{Conclusion}

This study demonstrated the performance of the Elecsys Anti-SARSCoV-2 S immunoassay, with a very high specificity of $99.95 \%$ (7876/ 7880 ) and sensitivity of $97.92 \%$ (235/240) in samples $\geq 14$ days postPCR confirmation, which was comparable to other commercially available immunoassays. Therefore, these data support the use of the Elecsys Anti-SARS-CoV-2 S immunoassay for reliable identification of past exposure to SARS-CoV-2 in various populations, and highlight the potential for the use of this assay in determining immune status during vaccine efficacy studies.

\section{Funding}

This study was funded by Roche Diagnostics GmbH (Mannheim, Germany). Roche Diagnostics GmbH (Mannheim, Germany) provided financial support for the study and for preparation of the article. Roche Diagnostics was involved in the study design; in the collection, analysis and interpretation of data; in the writing of the report; and in the decision to submit the article for publication.

\section{Data availability}

Qualified researchers may request access to individual patient level data through the clinical study data request platform (https://vivli. org/). Further details on Roche's criteria for eligible studies are available here: https://vivli.org/members/ourmembers/. For further details on Roche's Global Policy on the Sharing of Clinical Information and how to request access to related clinical study documents, see here: https://www.roche.com/research_and_development/who_we_ar e_how_we_work/clinical_trials/our_commitment_to_data_sharing.htm.

\section{Ethics approval}

The samples used in this study were collected and tested in accordance with applicable regulations, including relevant European Union directives and regulations, and the principles of the Declaration of Helsinki. All samples from Augsburg, Heidelberg, Berlin and Hagen were anonymized. A statement was obtained from the Ethics Committee (EC) of the Landesärztekammer Bayern confirming that there are no objections to the use of anonymized leftover samples. From the EC at the study site in Bern (Switzerland) a waiver was received and from the internal EC at the study site in Innsbruck (Austria) an approval was received. For Regensburg (Germany), EC approvals were already in place, amendments were submitted to notify the EC about Elecsys AntiSARS-CoV-2 $\mathrm{S}$ testing.

\section{Declaration of Competing Interest}

ER and AA report grants from Roche Diagnostics, during the conduct of the study and personal fees from Roche Diagnostics (honoraria for lectures), outside the submitted work. MK reports non-financial support from Roche Diagnostics, during the conduct of the study. CN, PF and JKH have nothing to disclose. FL, CMR, and TL are employees of Roche Diagnostics GmbH.

\section{Acknowledgments}

The authors would like to acknowledge: Kathrin Schoenfeld (Roche Diagnostics) for her role in study conceptualization, study management, interpretation of analysis, and further critical input; Michael Laimighofer (Roche Diagnostics) for his role in database generation and data validation, statistical analysis plan, and formal analysis; and Sigrid Reichhuber and Janina Edion (Roche Diagnostics) for their role in investigational site management, data acquisition, and study monitoring. This study was funded by Roche Diagnostics GmbH (Mannheim, Germany). Medical writing support was provided by Katie Farrant, Elements Communications Ltd, Westerham, UK and was funded by Roche Diagnostics International Ltd, Rotkreuz, Switzerland. COBAS, COBAS E, and ELECSYS are trademarks of Roche. All other product names and trademarks are the property of their respective owners.

\section{Appendix A. Supplementary data}

Supplementary material related to this article can be found, in the online version, at doi:https://doi.org/10.1016/j.jviromet.2021.114271.

\section{References}

Abbott, 2021. SARS-CoV-2 IgG Assay Method Sheet. Available from: https://www.fda gov/media/137383/download. Accessed July 282021.

Burbelo, P.D., Riedo, F.X., Morishima, C., Rawlings, S., Smith, D., Das, S., et al., 2020. Sensitivity in detection of antibodies to nucleocapsid and spike proteins of severe acute respiratory syndrome coronavirus 2 in patients with coronavirus disease 2019 . J. Infect. Dis. 222, 206-213. https://doi.org/10.1093/infdis/jiaa273.

Callegaro, A., Borleri, D., Farina, C., Napolitano, G., Valenti, D., Rizzi, M., et al., 2021. Antibody response to SARS-CoV-2 vaccination is extremely vivacious in subjects with previous SARS-CoV-2 infection. J. Med. Virol. 93, 4612-4615. https://doi.org/ 10.1002/jmv.26982.

Cavalcanti, E., Isgrò, M.A., Rea, D., Di Capua, L., Trillò, G., Russo, L., et al., 2021. Vaccination strategy and anti - SARS-CoV-2 S titers in healthcare workers of the INT IRCCS "Fondazione Pascale" Cancer center (Naples, Italy). Infect. Agent Cancer 16, 32. https://doi.org/10.1186/s13027-021-00375-2.

Chan, J.F., Yuan, S., Kok, K.H., To, K.K., Chu, H., Yang, J., et al., 2020. A familial cluster of pneumonia associated with the 2019 novel coronavirus indicating person-toperson transmission: a study of a family cluster. Lancet 395, 514-523. https://doi. org/10.1016/S0140-6736(20)30154-9.

Dai, L., Gao, G.F., 2021. Viral targets for vaccines against COVID-19. Nat. Rev. Immunol. 21, 73-82. https://doi.org/10.1038/s41577-020-00480-0.

Dashraath, P., Wong, J.L.J., Lim, M.X.K., Lim, L.M., Li, S., Biswas, A., et al., 2020. Coronavirus disease 2019 (COVID-19) pandemic and pregnancy. Am. J. Obstet. Gynecol. 222, 521-531. https://doi.org/10.1016/j.ajog.2020.03.021.

DiaSorin, 2020. LIAISON® SARS-CoV-2 S1/S2 IgG Assay Method Sheet; M0870004366 04/20. Available from: https://www.diasorin.com/sites/default/files/allegati/li aisonr_sars-cov-2_s1s2_igg_brochure.pdf.pdf. Accessed July 092021.

Ernst, E., Wolfe, P., Stahura, C., Edwards, K.A., 2021. Technical considerations to development of serological tests for SARS-CoV-2. Talanta 224, 121883. https://doi. org/10.1016/j.talanta.2020.121883.

EUROIMMUN, 2020a. Anti-SARS-CoV-2 ELISA (IgG) Product Data Sheet; EI_2606_D_UK_ A06, 10/2020. Available from: https://www.coronavirus-diagnostics.com/documen ts/Indications/Infections/Coronavirus/EI_2606_D_UK_A.pdf. Accessed July 092021.

EUROIMMUN, 2020b. Anti-SARS-CoV-2 ELISA (IgA) Product Data Sheet; EI_2606_D_UK_ B04, 10/2020. Available from: https://www.coronavirus-diagnostics.com/documen ts/Indications/Infections/Coronavirus/EI_2606_D_UK_B.pdf. Accessed July 092021. 
Farnsworth, C.W., Anderson, N.W., 2020. SARS-CoV-2 serology: much hype, little data. Clin. Chem. 66, 875-877. https://doi.org/10.1093/clinchem/hvaa107.

Forni, G., Mantovani, A., 2021. COVID-19 vaccines: where we stand and challenges ahead. Cell Death Differ. 28, 626-639. https://doi.org/10.1038/s41418-020-00720-

Galipeau, Y., Greig, M., Liu, G., Driedger, M., Langlois, M.A., 2020. Humoral responses and serological assays in SARS-CoV-2 infections. Front. Immunol. 11, 610688 https://doi.org/10.3389/fimmu.2020.610688.

Gavi, 2021. The COVID-19 Vaccine Race - Weekly Update. Available from: https://www. gavi.org/vaccineswork/covid-19-vaccine-race. Accessed August 042021.

Guo, L., Ren, L., Yang, S., Xiao, M., Chang, Yang F., et al., 2020. Profiling early humoral response to diagnose novel coronavirus disease (COVID-19). Clin. Infect. Dis. 71, 778-785. https://doi.org/10.1093/cid/ciaa310.

Hajian-Tilaki, K., 2014. Sample size estimation in diagnostic test studies of biomedical informatics. J. Biomed. Inform. 48, 193-204. https://doi.org/10.1016/j. jbi.2014.02.013.

Herishanu, Y., Avivi, I., Aharon, A., Shefer, G., Levi, S., Bronstein, Y., et al., 2021. Efficacy of the BNT162b2 mRNA COVID-19 vaccine in patients with chronic lymphocytic leukemia. Blood 137, 3165-3173. https://doi.org/10.1182/ blood.2021011568.

Johnson, M., Wagstaffe, H.R., Gilmour, K.C., Mai, A.L., Lewis, J., Hunt, A., et al., 2020 Evaluation of a novel multiplexed assay for determining IgG levels and functional activity to SARS-CoV-2. J. Clin. Virol. 130, 104572 https://doi.org/10.1016/j. jcv.2020.104572.

Kennedy, N.A., Lin, S., Goodhand, J.R., Chanchlani, N., Hamilton, B., Bewshea, C., et al., 2021. Infliximab is associated with attenuated immunogenicity to BNT162b2 and ChAdOx1 nCoV-19 SARS-CoV-2 vaccines in patients with IBD. Gut Epub ahead of print. https://doi.org/10.1136/gutjnl-2021-324789.

Kohmer, N., Westhaus, S., Ruhl, C., Ciesek, S., Rabenau, H.F., 2020. Brief clinical evaluation of six high-throughput SARS-CoV-2 IgG antibody assays. J. Clin. Virol. 129, 104480 https://doi.org/10.1016/j.jcv.2020.104480.

Kontou, P.I., Braliou, G.G., Dimou, N.L., Nikolopoulos, G., Bagos, P.G., 2020. Antibody tests in detecting SARS-CoV-2 infection: a meta-analysis. Diagnostics Basel (Basel) 10, 319. https://doi.org/10.3390/diagnostics10050319.

Long, Q.X., Liu, B.Z., Deng, H.J., Wu, G.C., Deng, K., Chen, Y.K., et al., 2020. Antibody responses to SARS-CoV-2 in patients with COVID-19. Nat. Med. 26, 845-848. https://doi.org/10.1038/s41591-020-0897-1.

Lou, B., Li, T.D., Zheng, S.F., Su, Y.Y., Li, Z.Y., Liu, W., et al., 2020. Serology characteristics of SARS-CoV-2 infection after exposure and post-symptom onset. Eur Respir. J. 56, 2000763 https://doi.org/10.1183/13993003.00763-2020.

Meyer, B., Torriani, G., Yerly, S., Mazza, L., Calame, A., Arm-Vernez, I., et al., 2020. Validation of a commercially available SARS-CoV-2 serological immunoassay. Clin. Microbiol. Infect. 26, 1386-1394. https://doi.org/10.1016/j.cmi.2020.06.024.

Muench, P., Jochum, S., Wenderoth, V., Ofenloch-Haehnle, B., Hombach, M., Strobl, M., et al., 2020. Development and validation of the Elecsys Anti-SARS-CoV-2 immunoassay as a highly specific tool for determining past exposure to SARS-CoV-2. J. Clin. Microbiol. 58 https://doi.org/10.1128/JCM.01694-20 e01694-01620.

Naqvi, A.A.T., Fatima, K., Mohammad, T., Fatima, U., Singh, I.K., Singh, A., et al., 2020 Insights into SARS-CoV-2 genome, structure, evolution, pathogenesis and therapies: structural genomics approach. Biochim. Biophys. Acta Mol. Basis Dis. 1866, 165878. https://doi.org/10.1016/j.bbadis.2020.165878.

Ni, L., Ye, F., Cheng, M.L., Feng, Y., Deng, Y.Q., Zhao, H., et al., 2020. Detection of SARSCoV-2-specific humoral and cellular immunity in COVID-19 convalescent individuals. Immunity 52 (971), 971-977. https://doi.org/10.1016/j. immuni.2020.04.023 e973.

Ocmant, A., Roisin, S., De Meuter, R., Brauner, J., 2021. Clinical performance of the Advia Centaur anti-SARS-CoV-2 chemiluminescent immunoassay related to antibody kinetics. J. Med. Virol. 93, 2583-2584. https://doi.org/10.1002/jmv.26800.

Okba, N.M.A., Muller, M.A., Li, W., Wang, C., GeurtsvanKessel, C.H., Corman, V.M., et al., 2020. Severe acute respiratory syndrome coronavirus 2-specific antibody responses in coronavirus disease patients. Emerg. Infect. Dis. 26, 1478-1488. https://doi.org/10.3201/eid2607.200841.

Ou, X., Liu, Y., Lei, X., Li, P., Mi, D., Ren, L., et al., 2020. Characterization of spike glycoprotein of SARS-CoV-2 on virus entry and its immune cross-reactivity with SARS-CoV. Nat. Commun. 11, 1620. https://doi.org/10.1038/s41467-020-15562-9.

Oved, K., Olmer, L., Shemer-Avni, Y., Wolf, T., Supino-Rosin, L., Prajgrod, G., et al., 2020. Multi-center nationwide comparison of seven serology assays reveals a SARS CoV-2 non-responding seronegative subpopulation. EClinicalMedicine 29, 100651. https://doi.org/10.1016/j.eclinm.2020.100651.

Premkumar, L., Segovia-Chumbez, B., Jadi, R., Martinez, D.R., Raut, R., Markmann, A., et al., 2020. The receptor binding domain of the viral spike protein is an immunodominant and highly specific target of antibodies in SARS-CoV-2 patients. Sci. Immunol. 5 https://doi.org/10.1126/sciimmunol.abc8413 eabc8413.

R Core Team, 2017. R: a Language and Environment for Statistical Computing. R Foundation for Statistical Computing, Vienna, Austria. Available from: https://www. R-project.org/. Accessed February 262021.

Resman Rus, K., Korva, M., Knap, N., Avšič Županc, T., Poljak, M., 2021. Performance of the rapid high-throughput automated electrochemiluminescence immunoassay targeting total antibodies to the SARS-CoV-2 spike protein receptor binding domain in comparison to the neutralization assay. J. Clin. Virol. 139, 104820 https://doi, org/10.1016/j.jcv.2021.104820.

Roche Diagnostics GmbH, 2021a. Elecsys ${ }^{\circledR}$ Anti-SARS-CoV-2 S Assay. Available from: htt ps://diagnostics.Roche.com/gb/en/products/params/elecsys-anti-sars-cov-2-s.html. Accessed July 092021

Roche Diagnostics GmbH, 2021b. Elecsys ${ }^{\circledR}$ Anti-SARS-CoV-2 Assay Method Sheet; 09203095501 V9.0. Available from: https://www.fda.gov/media/137605 /download. Accessed July 282021.

Roche Diagnostics GmbH, 2021c. Elecsys ${ }^{\circledR}$ Anti-SARS-CoV-2 S Assay Method Sheet, 09289267501 V2.0. Available from: https://www.fda.gov/media/144037 /download. Accessed July 092021.

Rubio-Acero, R., Castelletti, N., Fingerle, V., Olbrich, L., Bakuli, A., Wölfel, R., et al., 2021. In search of the SARS-CoV-2 protection correlate: head-to-head comparison of two quantitative S1 assays in pre-characterized oligo-/asymptomatic patients. Infect. Dis. Ther. 1-14. https://doi.org/10.1007/s40121-021-00475-x.

Salvagno, G.L., Henry, B.M., di Piazza, G., Pighi, L., De Nitto, S., Bragantini, D., et al., 2021. Anti-SARS-CoV-2 receptor-binding domain total antibodies response in seropositive and seronegative healthcare workers undergoing COVID-19 mRNA BNT162b2 vaccination. Diagnostics (Basel) 11, 832. https://doi.org/10.3390/ diagnostics11050832.

Seyahi, E., Bakhdiyarli, G., Oztas, M., Kuskucu, M.A., Tok, Y., Sut, N., et al., 2021. Antibody response to inactivated COVID-19 vaccine (CoronaVac) in immunemediated diseases: a controlled study among hospital workers and elderly. Rheumatol. Int. 41, 1429-1440. https://doi.org/10.1007/s00296-021-04910-7.

Shenzhen YHLO Biotech Co Ltd, 2020. iFlash-SARS-CoV-2 IgM/IgG Antibody Test. Available from: http://en.szyhlo.com/column/65/. Accessed July 282021.

Siemens, 2020. ADVIA Centaur® SARS-CoV-2 Total (COV2T) Assay Method Sheet; 11206904_EN Rev. 01, 2020-05. Available from: https://www.fda.gov/me dia/138446/download. Accessed July 092021.

Tang, T., Bidon, M., Jaimes, J.A., Whittaker, G.R., Daniel, S., 2020. Coronavirus membrane fusion mechanism offers a potential target for antiviral development. Antiviral Res. 178, 104792 https://doi.org/10.1016/j.antiviral.2020.104792.

To, K.K., Tsang, O.T., Leung, W.S., Tam, A.R., Wu, T.C., Lung, D.C., et al., 2020. Temporal profiles of viral load in posterior oropharyngeal saliva samples and serum antibody responses during infection by SARS-CoV-2: an observational cohort study. Lancet Infect. Dis. 20, 565-574. https://doi.org/10.1016/S1473-3099(20)30196-1.

Van Elslande, J., Decru, B., Jonckheere, S., Van Wijngaerden, E., Houben, E., Vandecandelaere, P., et al., 2020. Antibody response against SARS-CoV-2 spike protein and nucleoprotein evaluated by four automated immunoassays and three ELISAs. Clin. Microbiol. Infect. 26 (1557), e1551-e1557. https://doi.org/10.1016/j. cmi.2020.07.038.

Walls, A.C., Park, Y.J., Tortorici, M.A., Wall, A., McGuire, A.T., Veesler, D., 2020. Structure, function, and antigenicity of the SARS-CoV-2 spike glycoprotein. Cell 181, 281-292. https://doi.org/10.1016/j.cell.2020.02.058 e286.

Wang, Y., Zhang, L., Sang, L., Ye, F., Ruan, S., Zhong, B., et al., 2020. Kinetics of viral load and antibody response in relation to COVID-19 severity. J. Clin. Invest. 130, 5235-5244. https://doi.org/10.1172/JCI138759.

Wenzel, D., Zapf, A., 2013. Difference of two dependent sensitivities and specificities: comparison of various approaches. Biom. J. 55, 705-718. https://doi.org/10.1002/ bimj. 201200186.

Widge, A.T., Rouphael, N.G., Jackson, L.A., Anderson, E.J., Roberts, P.C., Makhene, M., et al., 2021. Durability of responses after SARS-CoV-2 mRNA-1273 vaccination. N. Engl. J. Med. 384, 80-82. https://doi.org/10.1056/NEJMc2032195.

World Health Organization, 2020. WHO Director-General's Remarks at the Media Briefing on 2019-nCoV on 11 February 2020. Available from: https://www.who.int /dg/speeches/detail/who-director-general-s-remarks-at-the-media-briefing-on2019-ncov-on-11-february-2020. Accessed July 072021.

World Health Organization, 2021. The COVID-19 Candidate Vaccine Landscape. Available from: https://www.who.int/publications/m/item/draft-landscape-of-covi d-19-candidate-vaccines. Accessed August 042021.

Wrapp, D., Wang, N., Corbett, K.S., Goldsmith, J.A., Hsieh, C.L., Abiona, O., et al., 2020. Cryo-EM structure of the 2019-nCoV spike in the prefusion conformation. Science 367, 1260-1263. https://doi.org/10.1126/science.abb2507.

Wu, F., Zhao, S., Yu, B., Chen, Y.M., Wang, W., Song, Z.G., et al., 2020. A new coronavirus associated with human respiratory disease in China. Nature 579, 265-269. https://doi.org/10.1038/s41586-020-2008-3.

Young, B.E., Ong, S.W.X., Ng, L.F.P., Anderson, D.E., Chia, W.N., Chia, P.Y., et al., 2020. Viral dynamics and immune correlates of COVID-19 disease severity. Clin. Infect. Dis. https://doi.org/10.1093/cid/ciaa1280. Aug 28 [Epub ahead of print].

Zhao, J., Yuan, Q., Wang, H., Liu, W., Liao, X., Su, Y., et al., 2020. Antibody responses to SARS-CoV-2 in patients with novel coronavirus disease 2019. Clin. Infect. Dis. 71 2027-2034. https://doi.org/10.1093/cid/ciaa344.

Zhu, F.C., Guan, X.H., Li, Y.H., Huang, J.Y., Jiang, T., Hou, L.H., et al., 2020. Immunogenicity and safety of a recombinant adenovirus type-5-vectored COVID-19 vaccine in healthy adults aged 18 years or older: a randomised, double-blind, placebo-controlled, phase 2 trial. Lancet 396, 479-488. https://doi.org/10.1016/ s0140-6736(20)31605-6. 